\title{
The Effects of SARS-CoV-2 Pandemic Countermeasures on Patients Receiving Infiltrative Treatment for Musculoskeletal Disorders: a Study from an Italian Cohort
}

\author{
D. Tarantino, R. Gnasso, F. Sirico, B. Corrado \\ Department of Public Health, University Federico II of Naples, Naples, Italy
}

\section{CORRESPONDING AUTHOR: \\ Domiziano Tarantino \\ Department of Public Health \\ University Federico II of Naples \\ via Sergio Pansini 5 \\ 80131 Naples, Italy \\ E-mail: domiziano22@gmail.com}

DOI:

10.32098/mltj.04.2021.09

LEVEL OF EVIDENCE: 4

\begin{abstract}
SUMMARY
Background. COVID-19 outbreak had a massive worldwide impact and several countermeasures to contain its spread have been adopted, such as the interruption of non-urgent outpatient clinics. We wanted to describe the effects of the national lockdown on the well-being of a cohort of Italian patients with musculoskeletal disorders receiving infiltrative treatment.

Methods. 40 patients who received intra- or peri-articular treatment were surveyed using a structuralised questionnaire that assessed their well-being during the first national lockdown.

Results. 24 out of 40 patients $(60 \%)$ did not come back after the re-opening of the Clinic because they were afraid of the pandemic. Of these 24 patients, the $83.3 \%$ noticed increased pain and stiffness in the joint. Due to the lockdown, the patient's quality of life was reduced by $61.66 \pm 15.72 \%$. After the lockdown, for the $92 \%$ of patients, the infiltrative treatment was perceived as more important than before, the $83 \%$ of patients perceived long-term security of infiltrative therapy availability as very important, and the $72 \%$ of the patients perceived the lockdown as inadequate.

Conclusions. The infiltrative outpatient clinic's interruption was seen to significantly worsen the physical condition of subjects with musculoskeletal disorders, with an important increase in both articular pain and stiffness. Therefore, any kind of infiltrative treatment suspension or delay should be avoided.
\end{abstract}

\section{KEY WORDS}

Infiltrative treatment; musculoskeletal disorders; SARS-CoV-2; COVID-19; Coronavirus

\section{INTRODUCTION}

The COVID-19 (COronaVirus Disease-19) pandemic, caused by the SARS-CoV-2 (Severe Acute Respiratory Syndrome CoronaVirus 2), had a massive impact all across the world during 2020, and marked our lives in an indelible way, changing the reality we were used to (1).

Italy has been the first European country to be hit by the pandemic, and it has also been among the most affected in the world after China, with the highest number of reported cases in Europe during the first outbreak of the pandemic (2-4). Consequently, several countermeasures to control the COVID-19's spread were implemented, such as a general lockdown and the suspension of public hospitals' outpa- tient clinics (2). This interruption put under pressure almost all the healthcare sectors, such as the Physical and Rehabilitation Medicine (PRM) ones, and negatively influenced the access to care of patients affected by musculoskeletal disorders (such as osteoarthritis) who periodically receive intraor peri-articular infiltrative treatment.

The intra- and peri-articular infiltrative treatment were proven to be safe and effective (5-7), and they are widely used for the treatment of several musculoskeletal disorders, such as arthritides, tendinopathies and fasciopathies, that can cause significant pain and functional limitation in patients, reducing their quality of life (QoL) and impairing their activities of daily living (ADL). 
At our infiltrative outpatient Clinic, injections are usually practiced using corticosteroids, corticosteroids and anesthetics, hyaluronic acid, and collagen.

After the first outbreak of the pandemic, at the re-opening of the injection Clinic, infiltrative therapy was carried out following the guidelines launched by the I.S.Mu.L.T. (Italian Society of Muscles, Ligaments and Tendons), that highlighted the importance of selecting patients not affected by COVID-19 (for example recognizing some musculoskeletal symptoms such as fatigue, myalgia and arthralgia that have been related to the novel Coronavirus (8)), to practice injections by implementing all the most appropriate measures to protect healthcare workers and patients from contagion, and to guarantee the maximum sterility and safety during the injection procedure (9). Furthermore, several studies showed how corticosteroid injections are safe and could be performed during the pandemic since they are not associated with a higher infection rate compared to the general population (10-13).

Infiltrative therapy has short-term effects on articular pain and stiffness, so it must be cyclically repeated, approximately every 6-12 months (depending on the underlying condition and on the used drug).

For this reason, we hypothesized that the suspension of infiltrative therapy due to the COVID-19 countermeasures could have an important impact on the physical condition of patients with musculoskeletal disorders, causing the reduction of their autonomy in complying with the ADL.

Therefore, the aim of this study is to describe how the countermeasures for COVID-19 influenced the well-being of a cohort of Italian patients with musculoskeletal disorders receiving intra- or peri-articular infiltrative treatment.

\section{Countermeasures against Covid-19}

The President of the Italian Republic enacted a decree on the 23 February 2020 to contain the SARS-CoV-2 contagion, stating that "urgent measures on the containment and management of the epidemiological emergency due to COVID-19" were needed (14). Following this national ordinance, the Campania region (with Naples being its capital and biggest city), which was one of the first regions in Italy to adopt all the measures against the COVID-19 spread, promulgated a regional regulation on the 5 March 2020 that established the immediate interruption of all the non-urgent outpatient activities provided by all hospitals (both public and private) until the 18 March 2020 (15).

The University Hospital Federico II of Naples, where our intra- and peri-articular injection treatment outpatient Clinic is based, and where this study was conducted, followed the regional regulation of the 5 March 2020 and then prolonged the suspension of deferrable outpatient activities until the 3 May 2020 (15).

From the 5 May to 17 June 2020, despite the re-opening of some outpatient Clinics, our intra- and peri-articular infiltrative treatment's outpatient Clinic was not operative since patients rejected to immediately resume the infiltrative treatment, being afraid of an increased risk of SARS-CoV-2 infection in the hospital setting.

\section{MATERIALS AND METHODS}

\section{Design}

This study is a survey on the lockdown's effects due to the SARS-CoV-2 pandemic on patients receiving intra- or peri-articular injections therapy. The survey is based on telephone interviews using a structuralised questionnaire (table I) like the one created by Dressler and Adib Saberi to assess the reduction of the quality of life of patients receiving botulinum toxin therapy during the first outbreak of the COVID-19 pandemic (16). Their questionnaire was modified to the aims of our study. The telephone calls were made in May 2021. The study meets the ethical standards of the journal (17). This study was carried out following the guidelines given by the local ethics committee. All information from patients were treated anonymously, and all data were saved on a laptop which access password was given just to the authors involved in the study.

\section{Treatment institution}

The study was performed at the Rehabilitation Unit, Department of Public Health, University of Naples Federi-

Table I. Structuralised questionnaire to survey the effects of the anti-coronavirus lockdown on patients receiving infiltrative treatment.

1. Increased articular pain: YES, NO

2. Increased articular stiffness: YES, NO

3. Reduction of quality of life (from 0 to $100 \%$ ):

4. Change of perception of the infiltrative treatment caused by lockdown: MORE IMPORTANT THAN BEFORE, NO CHANGE. IN PERCEPTION, LESS IMPORTANT THAN BEFORE

5. Perception of long-term security of the infiltrative treatment: IMPORTANT, VERY IMPORTANT, LESS IMPORTANT 6. Perception of the anti-coronavirus lockdown: ADEQUATE, NOT ADEQUATE 
co II, Naples, Italy. Two Resident Doctors (D.T., R.G.) and two Professors of Medicine (F.S., B.C.) were involved in the study. In 2019, a total of 394 intra- or peri-articular injections were performed, while in 2020, due to the lockdown and the subsequent closure of the infiltrative treatment's outpatient Clinic, only 182 injections were performed.

\section{Patients}

Patients were retrospectively collected from the Department's database. A total of 40 patients were recruited for this study. The age range was $42-82$ years. The inclusion criteria were the following: 1) patients who came once for treatment from October 2019 to the beginning of the first lockdown (8 March 2020), but then did not come back to the Clinic after its re-opening; 2) patients who cyclically came to the Clinic in 2019, but then did not return in 2020 at all; 3) patients who cyclically came to the Clinic from 2019 to the beginning of the first lockdown (8 March 2020) and then returned in 2020 but after a while since the re-opening of the Clinic.

The first inclusion criteria were established from October 2019 to the beginning of the first lockdown because it was a 6-month interval, so it was reasonable that patients who had their first cycle of treatment during that period, could then come back during the lockdown period.

The participation of patients in this study was entirely voluntary, and none of the invited patients declined their participation.

\section{Intra- or peri-articular treatment}

Intra- or peri-articular infiltrative treatments were performed using corticosteroids, corticosteroids and anaesthetics, collagen and hyaluronic acid. The musculoskeletal conditions treated were shoulder arthritis, rotator cuff tendinopathy, partial rotator cuff tear, epicondylitis, wrist arthritis, sacroiliac joint arthritis, hip arthritis, knee arthritis, Achilles tendinopathy, and plantar fasciitis.

Inter-injection intervals were usually settled between 6 to 12 months, and the choice of the most appropriate drug was tailored on patient's needs.

For the treatment of the arthritides, corticosteroids, corticosteroids plus anaesthetics, and hyaluronic acid were used. For the treatment of tendinopathies and fasciopathies, collagen was used.

Table II and table III report the treated anatomical regions, and how many intra-or peri-articular injections (and related used drug) were performed in 2019 and 2020, while in in table IV patient demographics are reported.

\section{RESULTS}

A summary of the results of the present survey is reported in table $\mathbf{V}$.

Of the 40 patients selected for the study, the $5 \%$ did not answer the telephone. The $20 \%$ of patients said that they did not come back after the re-opening of the Clinic since they felt good after the first cycle of treatment. The $15 \%$ of patients did not have any benefits after the intra- or peri-articular infiltrative treatment, so they did not want to come back to the Clinic.

24 of 40 patients (60\%) did not come back after the re-opening of the Clinic because they were afraid of the COVID19 pandemic.

For the $83.3 \%$ of patients who did not come back to the Clinic, pain and stiffness in the joint increased, for the $8.3 \%$ only pain increased (but not stiffness), and for another $8.3 \%$ only stiffness increased (but not pain).

Due to the lockdown, the patient's quality of life was reduced by $61.66 \pm 15.72 \%$ (range $30 \%-80 \%$ ).

Table II. Anatomical regions treated and number of injections performed in 2019.

- Shoulder: 14 corticosteroid and anaesthetic, 20 corticosteroid, 6 hyaluronic acid, 128 collagen

- Sacroiliac joint: 4 corticosteroid and anaesthetic

- Hip: 2 corticosteroid, 50 hyaluronic acid

- Knee: 16 corticosteroid and anaesthetic, 18 corticosteroid, 122 hyaluronic acid

- $\quad$ Foot: 14 collagen (8 for plantar fasciitis, 6 for Achilles tendinopathy)

Table III. Anatomical regions treated and number of injections performed in 2020.

- Shoulder: 16 corticosteroid and anaesthetic, 22 corticosteroid, 16 hyaluronic acid, 30 collagen

- Elbow: 6 collagen (for epicondylitis)

- Wrist: 12 corticosteroid and anaesthetic (for the trapeziometacarpal joint)

- Sacroiliac joint: 6 corticosteroid and anaesthetic

- Hip: 6 corticosteroid and anaesthetic, 8 hyaluronic acid

- Knee: 4 corticosteroid and anaesthetic, 44 hyaluronic acid

- Foot: 10 collagen (for Achilles tendinopathy) 
Table IV. Patient demographics and administered doses.

\begin{tabular}{lc}
\hline Total number of patients [n] & 40 \\
Male patients [n] & 28 \\
Female patients [n] & 12 \\
Patient age in 2021 (mean \pm standard deviation) [years] & $64.55 \pm 11.62$ \\
Injections performed in 2019 [n] & 394 \\
Injections performed in 2020 [n] & 182 \\
Reduction in performed injections between 2019 and $2020[\%]$ & 54
\end{tabular}

After the lockdown, for the $92 \%$ of patients, the infiltrative treatment was perceived as more important than before, while for the $8 \%$ there was no change in perception.

The $83 \%$ of patients perceived long-term security of infiltrative therapy availability as very important, $17 \%$ as important and none as less important.

$72 \%$ of the patients perceived the lockdown as inadequate, while the $28 \%$ perceived the lockdown as adequate.

\section{DISCUSSION AND CONCLUSIONS}

COVID-19 pandemic caused the interruption of hospitals' outpatient clinics in order to contain its spread, especially among the most fragile patients. The COVID19 pandemic had a negative effect on the care of patients with musculoskeletal disorders, since it is well known that all chronic musculoskeletal conditions (especially osteoarthritis) require a regular and accurate follow-up (18).

PRM clinical activities were heavily affected, negatively influencing the access to care of subjects who receive intra- or peri-articular infiltrative treatment.

Consequently, patients' quality of life during the lockdown period was reduced by $61.66 \pm 15.72 \%$. In the $92 \%$ of patients, the lockdown confirmed the perception of the importance of infiltrative treatment. The $83 \%$ of

Table V. Effects of the infiltrative treatment outpatient Clinic's lockdown on patients according to the administered questionnaire.

Symptoms caused by lockdown

- Increased joint pain [\% of patients] $\quad 8.3$

- Increased joint stiffness [\% of patients] $\quad 8.3$

- Increased both of them [\% of patients] 13.3

Reduction of quality of life caused by lockdown (mean \pm standard deviation) [\%] $\quad 61.66 \pm 15.72$

Change of perception of intra-articular treatment caused by lockdown

- Therapy is more important than before [\% of patients] 92

- No change [\% of patients] $1 \%$

- Therapy is less important than before [\% of patients] 0

Perception of long-term intra-articular treatment security

- Very important [\% of patients] 13

- Important [\% of patients] 17

- Less important [\% of patients] 0

Perception of lockdown

- Inadequate [\% of patients] $\quad 72$

- Adequate [\% of patients] 28 
patients felt the long-term infiltrative treatment security as very important for their health condition, and the $72 \%$ reported that their patient rights were not respected during the lockdown. Therefore, the pandemic and the subsequent interruption of intra- or peri-articular infiltrative therapy for patients with musculoskeletal diseases caused a worsening of their physical conditions, with a marked increase in articular pain and stiffness.

One limitation of our study is the relatively small sample and the lack of a control group, but it should be emphasized that, to our knowledge, this is the first survey that assessed the impact of COVID-19 countermeasures on patients receiving intra- or peri-articular infiltrative treatment.

\section{REFERENCES}

1. Giordano L, Cipollaro L, Migliorini F, Maffulli N. Impact of Covid-19 on undergraduate and residency training. Surgeon 2021;19(5):e199-e206.

2. Boldrini P, Bernetti A, Fiore P, SIMFER Executive Committee, SIMFER Committee for International Affairs. Impact of COVID-19 outbreak on rehabilitation services and Physical and Rehabilitation Medicine physicians' activities in Italy. An official document of the Italian PRM Society (SIMFER). Eur J Phys Rehabil Med 2020;56(3):316-8.

3. Tarantino D, Gnasso R, Migliore F, Iommazzo I, Sirico F, Corrado B. The effects of COVID-19 pandemic countermeasures on patients receiving botulinum toxin therapy and on their caregivers: a study from an Italian cohort. Neurol Sci 2021;42(8):3071-7.

4. Government of Italy. Decree of the President of the Council of Ministers 11 March 2020. Available at: https://www. gazzettaufficiale.it/eli/id/2020/03/11/20A01605/sg.

5. Honvo G, Reginster J-Y, Rannou F, et al. Safety of Intra-articular Hyaluronic Acid Injections in Osteoarthritis: Outcomes of a Systematic Review and Meta-Analysis. Drugs Aging 2019;36(1):101-27.

6. He W-W, Kuang M-J, Zhao J, et al. Efficacy and safety of intraarticular hyaluronic acid and corticosteroid for knee osteoarthritis: A meta-analysis. Int J Surg Lond Engl 2017;39:95-103.

7. Blaine T, Moskowitz R, Udell J, et al. Treatment of persistent shoulder pain with sodium hyaluronate: a randomized, controlled trial. A multicenter study. J Bone Joint Surg Am 2008;90(5):970-9.

8. Cipollaro L, Giordano L, Padulo J, Oliva F, Maffulli N. Musculoskeletal symptoms in SARS-CoV-2 (COVID-19) patients. J Orthop Surg 2020;15(1):178.

9. Oliva F, Vittadini F, Frizziero A, et al. I.S.Mu.L.T. Reccomendations for Intra and Periarticular Injections
In conclusion, the results of our study highlighted how the injections outpatient Clinic's interruption in 2020 worsened the physical condition of subjects with musculoskeletal disorders, causing them a considerable increase in their symptomatology. The importance of intra- and peri-articular infiltrative therapy to treat these patients and the need to administer it regularly and without any important interruption is essential to not lose its clinical benefits, so any kind of treatment suspension or delay must be absolutely avoided.

\section{CONFLICT OF INTERESTS}

The authors declare that they have no conflict of interests.

during COVID19 Pandemic. Muscle Ligaments Tendons J 2020;10(3):343.

10. Newton AC, Jones G, Jones JWM, Norris R, Barabas AG. Intra-articular corticosteroid injections during the COVID-19 lockdown period: A service evaluation. Musculoskeletal Care 2021;19(2):236-43.

11. Azwan Aziz M, Abu Hanifah R, Mohd Nahar AM. Musculoskeletal Corticosteroid Injection during COVID-19 Pandemic in Sabah: Is It Safe? Adv Orthop 2021;2021:8863210.

12. McKean D, Chung SL, Fairhead R, et al. Corticosteroid injections during the COVID-19 pandemic. Bone Jt Open 2020;1(9):605-11.

13. Chang CY, Prabhakar A, Staffa SJ, et al. Symptomatic COVID-19 infections in outpatient image-guided corticosteroid injection patients during the lockdown phase. Skeletal Radiol 2021;50(6):1117-23.

14. Government of Italy. Decree of the President of the Council of Ministers 23 February 2020. Available at: https:// www.gazzettaufficiale.it/eli/id/2020/02/23/20G00020/sg.

15. University of Naples Federico II Official Statement 6 March 2020. Available at: https://www.policlinico.unina. it/flex/cm/pages/ServeBLOB.php/L/IT/IDPagina/3234.

16. Dressler D, Adib Saberi F. Botulinum toxin therapy in the SARS-CoV-2 pandemic: patient perceptions from a German cohort. J Neural Transm (Vienna) 2020;127(9):1271-4.

17. Padulo J, Oliva F, Frizziero A, Maffulli N. Muscle Ligaments Tendons Journal - Basic principles and recommendations in clinical and field science research: 2018 update. Muscle Ligaments Tendons J 2018;8(3):305-7.

18. National Clinical Guideline Centre (UK). Osteoarthritis: Care and Management in Adults. London: National Institute for Health and Care Excellence (UK); 2014. 